\title{
Factors predicting early mortality after radical cystectomy for urothelial carcinoma in a contemporary cohort of patients
}

\author{
Sandra Kim ${ }^{1}$; Andrea Kokorovic ${ }^{1}$; Vidit Sharma ${ }^{2}$; Stephen Boorjian²; Ricardo A. Rendon ${ }^{1}$; \\ David Bell ${ }^{1}$; Ross Mason ${ }^{1}$ \\ ${ }^{1}$ Department of Urology, Dalhousie University, Halifax, NS; ${ }^{2}$ Mayo Clinic, Rochester, MN, United States
}

Support: This study was supported by the VA Health Services and Research Fellowship.

Cite as: Can Urol Assoc J 2020 June 16; Epub ahead of print. http://dx.doi.org/10.5489/cuaj.6400

Published online June 16, 2020

$* * *$

\begin{abstract}
Introduction: We aimed to identify preoperatively available patient variables associated with increased mortality within 30 and 90 days of radical cystectomy (RC) for localized urothelial carcinoma (UC), and to evaluate temporal trends in early mortality rates.
\end{abstract}

Methods: We reviewed the National Cancer Database to identify patients who underwent RC for UC between 2006 and 2013. Preoperatively available patient-specific demographics and mortality rates at 30 and 90 days postoperatively were analyzed. Univariable and multivariable logistic regression analyses were performed to examine factors associated with 30- and 90-day mortality.

Results: We identified 37366 patients who underwent RC between 2006 and 2013. Overall mortality rates remained stable over time. From 2006-2013, 936 patients $(2.5 \%)$ and 2554 patients $(6.8 \%)$ died of any cause within 30 and 90 days post-RC, respectively. On multivariable analysis, increased age, higher clinical $\mathrm{T}$ and $\mathrm{N}$ stage, increased Charlson-Deyo comorbidity classification, African-American race, lower hospital volume, non-academic centers, lower patient income, and absence of insurance were each significantly associated with increased early mortality after $\mathrm{RC}(\mathrm{p}<0.05)$. The protective effect of higher hospital volume was similar regardless of patient's age, clinical stage, or comorbidity status.

Conclusions: Our study identified patient-specific variables that are significantly associated with increased early mortality after RC. These findings can be used in counselling to identify ideal candidates for RC to decrease patient harm. Furthermore, early mortality rates after RC have remained stable over time, indicating that ongoing quality improvement is essential to improve outcomes. 


\section{Introduction}

Bladder cancer is one of the most common malignancies in North America. In the United States alone in 2018 there were approximately 81,190 new diagnoses of bladder cancer with 17,240 deaths attributed to the disease. ${ }^{1}$ Radical cystectomy (RC) is the gold standard treatment for patients with muscle-invasive bladder cancer (MIBC) and for those with non-MIBC who are unresponsive to intravesical therapy. ${ }^{2}$ There is a known high risk of perioperative morbidity and mortality given the complexity of RC and urinary diversion, especially in the elderly or frail populations. Variations exist in published early mortality rates, which have been estimated to be up to $20 \%$ in early studies, down to $1.1-2.7 \%$ in more recent literature, with an overall complication rate of approximately $34 \%{ }^{3-6}$ As life expectancy increases, the number of RCs offered is predicted to increase, particularly in elderly patients. ${ }^{7}$ Given the high risk of morbidity and mortality associated with RC, it is important to gain a more granular understanding of postoperative mortality to inform patients. Counselling and discussing the predicted risks and benefits of this treatment with patients in order to select appropriate candidates for this procedure will help reduce patient harm.

Although several previous studies have investigated post-operative mortality after RC and nomograms have been developed, many of these studies have focused on post-operative variables in their models. ${ }^{8-13}$ This limits their applicability to pre-operative clinical decision making and patient counseling. In addition, rates of change in early mortality over time have not been thoroughly investigated. Herein, we examine temporal trends in early mortality rates after $\mathrm{RC}$ and investigate pre-operatively available patient variables associated with early mortality.

\section{Methods}

\section{Data source}

The National Cancer Database (NCDB), a joint effort between the American Cancer Society and the American College of Surgeons Commission on Cancer, includes information from patients who received an initial diagnosis or first course of treatment for cancer at one of the nearly 1500 Commission on Cancer accredited cancer centers. ${ }^{14}$ The Commission on Cancer accredited centers are expected to maintain high-quality cancer registries, using trained personnel and standardized methodology to abstract clinical, pathologic, treatment, and demographic data. The dataset includes information from more than $70 \%$ of incident malignancies within the United States. ${ }^{14}$ All patient information has been de-identified and thus, the study's international review board approval for ethics was exempted.

\section{Study population}

We identified all patients in the NCDB who were diagnosed with urothelial carcinoma of the bladder (all variants) between 2006-2013. Patients diagnosed with non-urothelial histology were excluded. 


\section{Outcomes of interest}

The primary aim was to report on the 30- and 90- day mortality rates after RC for UC. The secondary aims were to examine clinical, demographic, and socioeconomic factors associated with 30- and 90- day mortality after RC.

\section{Statistical analysis}

Baseline clinical and demographic characteristics between patients who died or did not die within 30- and 90-days of RC were compared using the t-test for independent means for continuous variables and chi-square test for categorical variables. The proportion of patients who died each year within 30- and 90-days of RC were calculated, and trends were plotted by year. Multivariable logistic regression analyses were utilized to examine factors associated with 30and 90-day mortality. Variables for inclusion into the multivariable model were chosen clinically. Additionally, due to the well described association between hospital volume and mortality after radical cystectomy, we performed interaction analyses to determine whether this association was present across patients of different ages, comorbidity status, and cT stage. We hypothesized that the impact of hospital volume would be greater among older, more co-morbid patients with higher stage of disease.

Statistical analyses were performed using R version 3.3.2 (R Foundation for Statistical Computing, Vienna, Austria). All tests were two-sided, with p-values $<0.05$ considered statistically significant.

\section{Results}

\section{Baseline characteristics}

We identified 37,366 patients who underwent RC for UC between 2006-2013. The mean age was $67.85( \pm 10.39) ; 75.8 \%$ were male and $38.0 \%$ had cT2 disease. The majority of patients were Caucasian (91.1\%) and a Charlson-Deyo comorbidity index of 0 was noted in $79.6 \%$ of patients. Table 1 provides the baseline characteristics of all included patients.

\section{Temporal trends in mortality}

Between 2006 - 2013, 936 patients (2.5\%) and 2554 patients (6.8\%) died of any cause at 30- and 90 - days after RC, respectively. Over the study period, the 30 - and 90 -day mortality rates remained stable within the patient population captured in the NCDB.

\section{Factors associated with mortality}

Baseline differences between those who died and those who did not die are summarized in Table 2 and multivariable analysis of factors associated with increased mortality at 30- and 90- days post $\mathrm{RC}$ is demonstrated in Table 3. Increased age, higher clinical T stage, African-American race, and increased Charlson-Deyo comorbidity index were associated with increased mortality on multivariable analysis at both 30- and 90-days. Within the NCDB database dictionary, the 
Charlson-Deyo score was calculated excluding the patient's primary cancer, and the score is truncated so that a score of 2 is the maximum reported value. Receipt of neoadjuvant chemotherapy was associated with decreased mortality at both 30- and 90-days. Females were less likely to die at 30 days but not at 90 days. Lymph node metastases was associated with increased risk of death at 90 days but not at 30 days. Higher income status, hospital volume, and use of private insurance were associated with decreased mortality at 90 days post RC.

No interaction was identified in 90-day mortality between hospital volume and age, comorbidity status, or cT stage suggesting that the association between hospital volume and mortality is similar regardless of patient characteristics (all $\mathrm{p}>0.05$ ).

\section{Discussion}

Herein we examined trends in early mortality rates after $\mathrm{RC}$ for $\mathrm{UC}$ and explored factors associated with such. We identified that mortality rates remained similar at 30- and 90-days post RC from 2006 to 2013. In addition, several preoperative factors were identified as being associated with early mortality.

Given that our study population from the NCDB draws records from Commission on Cancer accredited cancer centers only, our study population may underestimate the total mortality rates if all centers, including non-accredited cancer centers performing RCs, were taken into account. When comparing the mortality rates amongst institutions by hospital volume of $\mathrm{RC}$, there was a clear difference in terms of hospital volume - with some centers performing less than ten RCs per year, and others performing close to a thousand RCs per year. Our study demonstrates that mortality post RC is decreased at 90-days at high volume centers. Previous studies have also demonstrated significant association between hospital and surgeon specific volume with morbidity and mortality post RC. ${ }^{15,16}$ Thus, the average mortality per year may be quite different depending on the type of institution and hospital volume. Our study additionally found that the protective effect of increasing hospital volume was present regardless of patient characteristics. These data argue for the centralization of radical cystectomy to higher volume centers.

Our study has identified several socioeconomic variables associated with increased mortality following RC. First, African-American race was associated with significant increase in risk of death at both 30 and 90-days postoperatively. This is in keeping with previously published literature, which suggested that black race is associated with increased length of hospital stay and inferior overall survival in a cohort of patients undergoing RC for MIBC. ${ }^{17}$ Previous work has shown that African Americans are less likely to receive care at high volume centers, thereby placing them at higher risk for post-operative complications and mortality. ${ }^{18}$ Second, we have identified that higher income level and availability of private insurance were associated with decreased mortality following $\mathrm{RC}$, which is also in keeping with previously published literature. ${ }^{19}$ The results of our study highlight an ongoing and unmet need to decrease current gaps in racial and socioeconomic disparities observed in patients with bladder cancer. 
The baseline characteristics of our study population who underwent RC were consistent with current literature, which demonstrates that patients who are younger, have fewer comorbidities, and localized disease are more likely to undergo RC for management of their bladder cancer..$^{8-13,20-25}$ Our study also found that increased age, advanced clinical stage, increased comorbidity, and decreased hospital volume were significantly associated with increased mortality as indicated in Table 3. Interestingly, we identified a significantly decreased mortality among patients receiving neoadjuvant chemotherapy (NAC) prior to RC at both 30and 90- days after RC. As it is unlikely that advantages in early mortality from NAC would be seen this soon after $\mathrm{RC}$, this may simply reflect a selection bias with healthier patients receiving NAC. ${ }^{26,27}$ Recent studies have demonstrated that use of NAC does not increase early postoperative complications or mortality rates, and therefore our mortality data also supports routine use of NAC in patients undergoing RC for MIBC. ${ }^{28}$

Our study demonstrates relatively stable temporal trends in post cystectomy mortality rates between 2006-2013. This suggests that there remain significant gains to be made with respect to quality improvement and patient survival. Recently, there has been increased awareness and implementation of RC-specific enhanced recovery after surgery (ERAS) protocols with promising outcomes. Recent systemic review and meta-analyses of patient outcomes post RC who underwent ERAS protocols demonstrated a shorter time for bowel recovery, decreased hospital stay, as well as lower rates of post-operative complications, such as ileus and cardiovascular complications. ${ }^{29,30}$ Based on the data published herein, it will be interesting and imperative to investigate whether such protocols lead to improved early mortality as well. Other variables to consider in the future will include novel and emerging drug agents, specifically immunotherapy in the neoadjuvant setting, and their effects on post-operative morbidity and mortality.

One of the biggest benefits of using a national database such as the NCDB is the number of patients we were able to incorporate into our analysis. To date, our study is the largest published analysis looking into mortality associated with radical cystectomy with over 30,000 patients included in our analysis. However, we acknowledge several limitations to this study associated with using such a large database. First, as with all retrospective studies, the present analysis is limited by potential unmeasured effect modifiers and thus we can only identify associations and not causality. Second, the NCDB draws its data from a non-population based database, and presumably our study population could underestimate the actual observed mortality rates after RC in non-accredited cancer centers, or overestimate the mortality in accredited cancer centers of excellence with high volume of RC. The wide variances in the hospital volume of RC introduces the element of surgeon volume specific bias and differences in mortality, and this was not able to be accounted for in our study. Furthermore, high volume in this study was measured as $>20$ cases per year, which does not reflect true high-volume centers where RC numbers may approach the hundreds. Third, the population in this study was 
overwhelmingly Caucasian, and the effect sizes demonstrated in our study might not be applicable to other races that were not significantly represented in our population.

In addition, although the NCDB performs validation and data quality studies, there is nonetheless the possibility of errors within the database. Calculation of temporal trends rely on study populations remaining stable over time and this is another known limitation of using the NCDB for trend analysis. In our study, the number of hospital centers and cystectomies increased over time in the $\mathrm{NCDB}$, with no significant variation in early mortality after RC. Lastly, the use of a retrospective database for our study limits our ability to analyze the specific cause of death. We hypothesize that deaths in the early post-operative period at 30 - and 90-days post RC were related to the surgery itself.

The mortality rates from our study demonstrates important data for pre-operative patient counseling. These variables can be used to identify at-risk patients who are female, older, have higher Charlson-Deyo comorbidity indexes, or higher clinical T stage to facilitate candid discussions with patients. These discussions might help prevent inappropriate patient selection for this high-risk surgery thus reducing mortality in significantly comorbid patients who might be considering radical cystectomy as a therapy.

\section{Conclusions}

Early mortality after RC has remained relatively stable and significant over time. Our study demonstrates the importance of careful discussion and selection of patients for management of MIBC with RC by taking into account pre-existing patient variables. In addition, the stable mortality rate also highlights the need for ongoing quality improvement and national databases to monitor improvements in patient care and trends over time. High quality databases and ongoing research will help identify the best candidates or help optimize patients pre-operatively prior to their RC, reducing patient morbidity and mortality. 


\section{References}

1. Siegel RL, Miller KD, Jemal DVM A. Cancer statistics, 2018. CA Cancer J Clin 2018; 68:7-30.

2. Herr HW, Dota Z, Donat SM, et al. Defining optimal therapy for muscle invasive bladder cancer. J Urol 2007; 177:437-43.

3. Glantz GM. Cystectomy and urinary diversion. J Urol 1966; 96:714-17.

4. Bostrom PJ, Kossi J, Laato M, et al. Risk factors for mortality and morbidity related to radical cystectomy. BJU Int 2009; 103:191-96.

5. Coughlin GD, Youl PH, Philpot S, et al. Outcomes following radical cystectomy: a population-based study from Queensland. ANZ J Surg 2019; 89:752-57.

6. Djaladat H, Katebian B, Bazargani ST, et al. 90-Day complication rate in patients undergoing radical cystectomy with enhanced recover protocol: a prospective study. World J Urol 2017:35:907-11.

7. Rosario DJ, Becker M, Anderson JB. The changing pattern of mortality and morbidity from radical cystectomy. BJUI Int 2003; 85:427-30.

8. Isbarn $\mathrm{H}$, Jeldres $\mathrm{C}$, Zini L, et al. A population-based assessment of perioperative mortality after cystectomy for bladder cancer. J Urol 2009; 182:70-77.

9. Taylor JM, Feifer A, Savage CJ, et al. Evaluating the utility of a preoperative nomogram for predicting 90-day mortality following radical cystectomy for bladder cancer. BJU Int 2012; 109:855-59.

10. Aziz A, May M, Burger M, et al. Prediction of 90-day mortality after radical cystectomy for bladder cancer in a prospective European multicenter cohort. Eur Urol 2014; 66:15663.

11. Williams SB, Huo J, Chu Y, et al. Cancer and all-cause mortality in bladder cancer patients undergoing radical cystectomy: Development and validation of a nomogram for treatment decision-making. Urology 2017; 110:76-83.

12. Simone G, Bianchi M, Giannarelli D, et al. Development and external validation of nomograms predicting disease-free and cancer-specific survival after radical cystectomy. World J Urol 2015; 33:1419-28.

13. Kluth LA, Black PC, Bochner BH, et al. Prognostic and prediction tools in bladder cancer: A comprehensive review of the literature. Eur Urol 2015; 68:238-53.

14. Bilmoria KY, Stewart AK, Winchester DP, et al. The National Cancer Data Base: A powerful initiative to improve cancer care in the United States. Ann Surg Oncol 2008; 15:683-90.

15. Konety BR, Dhawan V, Allareddy V, et al. Impact of hospital and surgeon volume on inhospital mortality from radical cystectomy: data from the health care utilization project. $J$ Urol 2005; 173:1695-700.

16. Finks JF, Osborne NH, and Birkmeyer JD. Trends in hospital volume and operative mortality for high-risk surgery. N Engl J Med 2011; 364:2128-37.

17. Gild P, Wancowicz SA, Sood A et al. Racial disparity in quality of care and overall survival among black vs white patients with muscle invasive bladder cancer treated with radical cystectomy: A national cancer database analysis. Urol Oncol 2018; 36:e1-e11. 
18. Konety B, Allareddy V, Carroll P. Factors affecting outcomes after radical cystectomy in African Americans. Cancer 2007; 109:542-48.

19. Nazemi A, Ghodoussipour S, Pearce S et al. Socioeconomic and insurance status are independent prognostic indicators of higher disease stage and worse prognosis in bladder cancer. Urol Oncol 2019; 37:784-90.

20. Monzo Gardiner GI, Herranz Amo F, Diez Cordero JM, et al. Prognostic factors for survival in patients with transitional bladder cancer treated with radical cystectomy. Actas Urol Esp 2009; 33:249-57.

21. Elmussareh M, Simonsen PC, Young M, et al. Correlation between organ-specific comorbidities and complications in bladder cancer patients undergoing radical cystectomy. Scand J Urol 2018; 52:395-400.

22. Parikh N and Sharma P. Frailty as a prognostic indicator in the radical cystectomy population: a review. Int Urol Nephrol. 2019; 51:1281-90.

23. Froehner M, Koch R, Heberling U, et al. Which comorbidity classification is best suited to identify patients at risk for 90-day and long-term non-bladder cancer mortality after radical cystectomy? [published online ahead of print July 2, 2019] World J Urol 2019; doi:10.1007/s00345-019-02860-1.

24. Coughlin GD, Youl PH, Philpot S, et al. Outcomes following radical cystectomy: a population-based study from Queensland, Australia. Anz J Surg 2019; 89:752-57.

25. Pavone C, Candela L, Fontana D, et al. Postoperative complications and 90-day mortality in radical cystectomy in high-risk patients: A monocentric retrospective observational study. Urologia. 2018; 85:111-17.

26. Grossman HB, Natale RB, Tangen CM, et al. Neoadjuvant chemotherapy plus cystectomy compared with cystectomy alone for locally advanced bladder cancer. $N$ Eng J Med 2003; 349:859-66.

27. Collaboration ABC. Neoadjuvant chemotherapy in invasive bladder cancer: A systematic review and meta-analysis. Lancet 2003; 361:1927-34.

28. Milenkovic U, Akand M, Moris L et al. Impact of neoadjuvant chemotherapy on shortterm complications and survival following radical cystectomy. World J Urol 2019; 37:1857-66.

29. Xiao J, Wang M, He W, et al. Does postoperative rehabilitation for radical cystectomy call for enhanced recovery after surgery? A systemic review and meta-analysis. Curr Med Sci 2019; 39:99-110.

30. Giannarini G, Crestani A, Inferrera A, et al. Impact of Enhanced Recovery After Surgery (ERAS) protocol versus standard of care on perioperative outcomes of radical cystectomy: a systemic review and meta-analysis of comparative studies. Minerva Urol Nefrol 2019; 71:309-323. 


\section{Figures and Tables}

Fig. 1.

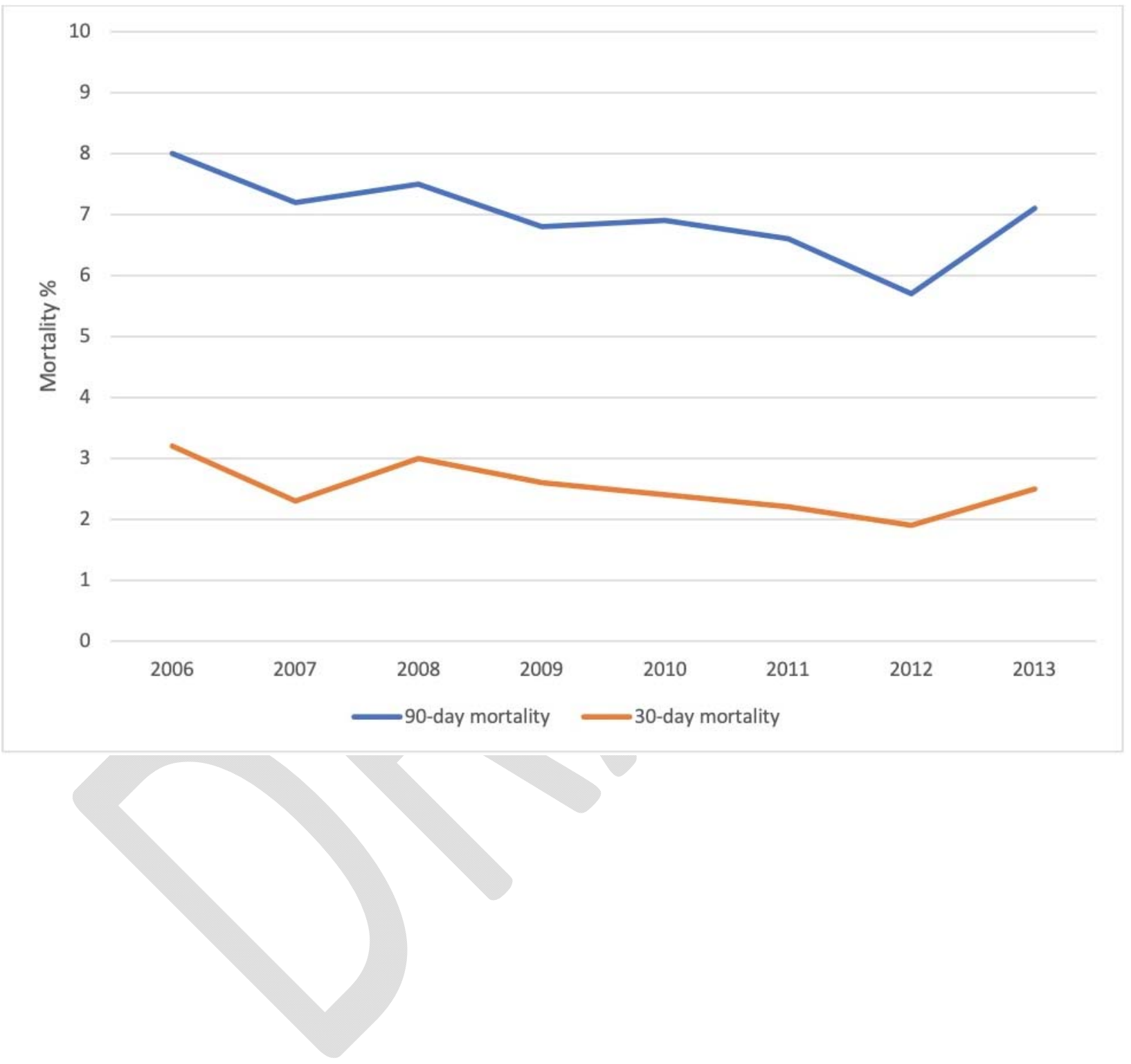




\begin{tabular}{|l|c|}
\hline \multicolumn{2}{|l|}{ Table 1. Baseline characteristics of the study population $(\mathbf{n}=\mathbf{3 7 3 6 6})$} \\
\hline Number of patients & 37,366 \\
\hline Mean age (SD) & $67.85(10.39)$ \\
\hline Female sex (\%) & $9056(24.2)$ \\
\hline Clinical stage (\%) & $4890(13.1)$ \\
\hline cT1 & $14187(38.0)$ \\
\hline cT2 & $2596(6.9)$ \\
\hline cT3 & $1931(5.2)$ \\
\hline cT4 & $838(2.2)$ \\
\hline cTa & $479(1.3)$ \\
\hline cTis & $12445(33.3)$ \\
\hline cT N/A & \\
\hline Clinical N stage (\%) & $25915(69.4)$ \\
\hline cN0 & $2148(5.7)$ \\
\hline cN1 & $9303(24.9)$ \\
\hline cNX & \\
\hline Clinical M stage (\%) & $35227(94.3)$ \\
\hline cM0 & $579(1.5)$ \\
\hline cM1 & $1560(4.2)$ \\
\hline cM N/A & $6352(17)$ \\
\hline Patients with neoadjuvant chemotherapy (\%) & \\
\hline Race (\%) & $34031(91.1)$ \\
\hline Caucasian & $2133(5.7)$ \\
\hline African-American & $818(2.2)$ \\
\hline Other & $384(1.0)$ \\
\hline N/A & $26381(70.6)$ \\
\hline Charlson-Deyo comorbidity index (\%) & $8423(22.5)$ \\
\hline 0 & $2562(6.9)$ \\
\hline 1 & $1092(2.9)$ \\
\hline 2 & $11859(31.7)$ \\
\hline Insurance (\%) & $1614(4.3)$ \\
\hline No insurance & $361(1.0)$ \\
\hline Private & $853(2.3)$ \\
\hline Medicaid & $21480(57.5)$ \\
\hline Medicare & \\
\hline Other government & \\
\hline N/A & \\
\hline Patient income (\%) & \\
\hline Low (\$<4600) & \\
\hline & \\
\hline
\end{tabular}




\begin{tabular}{|l|c|}
\hline High $(\$ \geq 46000)$ & $14478(38.7)$ \\
\hline N/A & $1408(3.8)$ \\
\hline Community (\%) & $28405(76.0)$ \\
\hline Metro & $5440(14.6)$ \\
\hline Urban & $2153(5.8)$ \\
\hline Rural & $1368(3.7)$ \\
\hline N/A & $17911(47.9)$ \\
\hline Hospital facility (\%) & $19213(51.4)$ \\
\hline Non-academic & $242(0.6)$ \\
\hline Academic & $20148(53.9)$ \\
\hline N/A & $6925(18.5)$ \\
\hline Hospital volume per year (\%) & $10293(27.5)$ \\
\hline$<10$ & \\
\hline $10-20$ & $5439(14.6)$ \\
\hline$>20$ & $31401(84.0)$ \\
\hline Education (\%) & $526(1.4)$ \\
\hline$>20 \%$ (lower education) & \\
\hline$<20 \%$ (higher education) & \\
\hline N/A &
\end{tabular}

"Education: Patient's measure of education was estimated by their area of residence by zip code and the proportion of adults in that zip code that did not graduate from high school from 20082012. N/A: not available; SD: standard deviation. 


\begin{tabular}{|c|c|c|c|c|c|c|}
\hline \multirow{2}{*}{ from $2006-2013(n=36738)$} & \multicolumn{3}{|c|}{30 days } & \multicolumn{2}{|c|}{90 days } & \multirow[b]{2}{*}{ p } \\
\hline & Alive & Dead & p & Alive & Dead & \\
\hline Number of patients & 36089 & 936 & 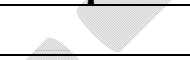 & 34184 & 2554 & \\
\hline Age (SD) & $67.69(10.37)$ & $73.69(9.36)$ & $<0.001$ & $67.50(10.34)$ & $72.48(9.86)$ & $<0.001$ \\
\hline Female sex $(\%)$ & $8754(24.3)$ & $222(23.7)$ & 0.733 & $8249(24.1)$ & $662(25.9)$ & 0.044 \\
\hline \multicolumn{3}{|l|}{ Clinical T stage (\%) } & 0.001 & \multicolumn{2}{|l|}{ 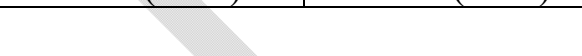 } & $<0.001$ \\
\hline cT1 & $4736(13.1)$ & $94(10.0)$ & +2 & $4566(13.4)$ & $224(8.8)$ & \\
\hline cT2 & $13728(38.0)$ & $343(36.6)$ & 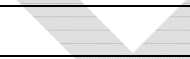 & $13109(38.3)$ & $851(33.3)$ & \\
\hline cT3 & $2505(6.9)$ & $69(7.4)$ & 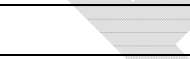 & $2334(6.8)$ & $227(8.9)$ & \\
\hline cT4 & $1848(5.1)$ & $68(7.3)$ & & $1651(4.8)$ & $252(9.9)$ & \\
\hline $\mathrm{cTa}$ & $809(2.2)$ & $16(1.7)$ & 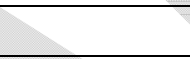 & $780(2.3)$ & $34(1.3)$ & \\
\hline cTis & $468(1.3)$ & $6(0.6)$ & 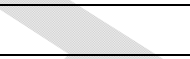 & $451(1.3)$ & $17(0.7)$ & \\
\hline $\mathrm{cT} \mathrm{N} / \mathrm{A}$ & $11995(33.2)$ & $340(36.3)$ & 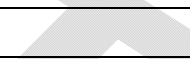 & $11293(33.0)$ & $949(37.2)$ & \\
\hline \multicolumn{3}{|l|}{ Clinical N stage (\%) } & $<0.001$ & \multicolumn{2}{|l|}{ 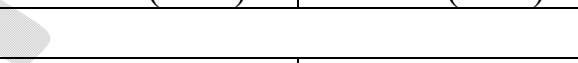 } & $<0.001$ \\
\hline $\mathrm{cNO}$ & $25096(69.5)$ & $585(62.5)$ & & $23915(70.0)$ & $1538(60.2)$ & \\
\hline $\mathrm{cN} 1$ & $2073(5.7)$ & $58(6.2)$ & & $1877(5.5)$ & $242(9.5)$ & \\
\hline $\mathrm{cNX}$ & $8920(24.7)$ & $293(31.3)$ & & $8392(24.5)$ & $774(30.3)$ & \\
\hline \multicolumn{3}{|l|}{ Clinical M stage (\%) } & 0.001 & & & $<0.001$ \\
\hline cM0 & $34054(94.4)$ & $866(92.5)$ & +2 & $32369(94.7)$ & $2286(89.5)$ & \\
\hline cM1 & $548(1.5)$ & $28(3.0)$ & & $420(1.2)$ & $153(6.0)$ & \\
\hline $\mathrm{cM} \mathrm{N} / \mathrm{A}$ & $1487(4.1)$ & $42(4.5)$ & & $1395(4.1)$ & $115(4.5)$ & \\
\hline Neoadjuvant chemotherapy (\%) & $6198(17.2)$ & $85(9.1)$ & $<0.001$ & $5910(17.3)$ & $312(12.2)$ & $<0.001$ \\
\hline \multicolumn{3}{|l|}{ Race (\%) } & 0.166 & & & 0.007 \\
\hline Caucasian & $32895(91.1)$ & $836(89.3)$ & & $31179(91.2)$ & $2310(90.4)$ & \\
\hline African-American & $2042(5.7)$ & $68(7.3)$ & & $1907(5.6)$ & $179(7.0)$ & \\
\hline Other & $784(2.2)$ & $20(2.1)$ & & $747(2.2)$ & $44(1.7)$ & \\
\hline N/A & $368(1.0)$ & $12(1.3)$ & & $351(1.0)$ & $21(0.8)$ & \\
\hline
\end{tabular}


Kim et al

Preoperative patient variables associated with increased mortality post-RC for UC

\begin{tabular}{|c|c|c|c|c|c|c|}
\hline \multicolumn{3}{|c|}{ Charlson-Deyo comorbidity index $(\%)$} & \multirow[t]{2}{*}{$<0.001$} & \multirow[b]{2}{*}{$24370(71.3)$} & \multirow[b]{2}{*}{$1547(60.6)$} & \multirow[t]{2}{*}{$<0.001$} \\
\hline 0 & $25564(70.8)$ & $556(59.4)$ & & & & \\
\hline 1 & $8091(22.4)$ & $267(28.5)$ & & $7572(22.2)$ & $719(28.2)$ & \\
\hline 2 & $2434(6.7)$ & $113(12.1)$ & & $2242(6.6)$ & $288(11.3)$ & \\
\hline \multicolumn{3}{|l|}{ Insurance $(\%)$} & $<0.001$ & & & $<0.001$ \\
\hline No insurance & $1068(3.0)$ & $11(1.2)$ & 2 & $1008(2.9)$ & $53(2.1)$ & \\
\hline Private & $11600(32.1)$ & $153(16.3)$ & $z$ & $11182(32.7)$ & $477(18.7)$ & \\
\hline Medicaid & $1565(4.3)$ & $29(3.1)$ & 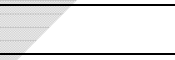 & $1462(4.3)$ & $115(4.5)$ & \\
\hline Medicare & $20678(57.3)$ & $720(76.9)$ & & $19400(56.8)$ & $1848(72.4)$ & \\
\hline Other government & $353(1.0)$ & $4(0.4)$ & 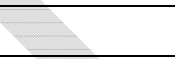 & $337(1.0)$ & $18(0.7)$ & \\
\hline $\mathrm{N} / \mathrm{A}$ & $825(2.3)$ & $19(2.0)$ & 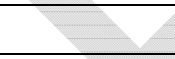 & $795(2.3)$ & $43(1.7)$ & \\
\hline \multicolumn{3}{|l|}{ Patient income $(\%)$} & 0.043 & & & $<0.001$ \\
\hline Low $(\$<46000)$ & $20733(57.4)$ & $562(60.0)$ & & $19571(57.3)$ & $1560(61.1)$ & \\
\hline High $(\$ \geq 46000)$ & $14007(38.8)$ & $330(35.3)$ & & $13353(39.1)$ & $877(34.3)$ & \\
\hline $\mathrm{N} / \mathrm{A}$ & $1349(3.7)$ & $44(4.7)$ & + & $1260(3.7)$ & $117(4.6)$ & \\
\hline \multicolumn{3}{|l|}{ Community (\%) } & 0.298 & \multicolumn{2}{|l|}{ 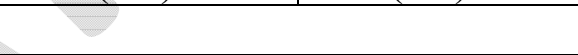 } & 0.004 \\
\hline Metro & $27423(76.0)$ & $713(76.2)$ & & $26006(76.1)$ & $1919(75.1)$ & \\
\hline Urban & $5269(14.6)$ & $131(14.0)$ & & $4985(14.6)$ & $377(14.8)$ & \\
\hline Rural & $2083(5.8)$ & $48(5.1)$ & & $1977(5.8)$ & $133(5.2)$ & \\
\hline $\mathrm{N} / \mathrm{A}$ & $1314(3.6)$ & $44(4.7)$ & 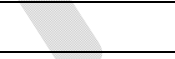 & $1216(3.6)$ & $125(4.9)$ & \\
\hline \multicolumn{3}{|l|}{ Hospital facility (\%) } & \multicolumn{3}{|l|}{0.001} & $<0.001$ \\
\hline Non-academic & $17253(47.8)$ & $505(54.0)$ & 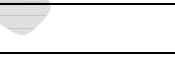 & $16325(47.8)$ & $1323(51.8)$ & \\
\hline Academic & $18599(51.5)$ & $428(45.7)$ & & $17632(51.6)$ & $1222(47.8)$ & \\
\hline $\mathrm{N} / \mathrm{A}$ & $237(0.7)$ & $3(0.3)$ & & $227(0.7)$ & $9(0.4)$ & \\
\hline Hospital volume per year (\%) & & & $<0.001$ & & & \\
\hline$<10$ & $19384(53.7)$ & $564(60.3)$ & & $18305(53.5)$ & $1502(58.8)$ & \\
\hline $10-20$ & $6707(18.6)$ & $164(17.5)$ & & $6373(18.6)$ & $452(17.7)$ & \\
\hline$>20$ & $9998(27.7)$ & $208(22.2)$ & & $9506(27.8)$ & $600(23.5)$ & \\
\hline \multicolumn{3}{|l|}{ Education $(\%)^{*}$} & 0.002 & & & $<0.001$ \\
\hline$>20$ (lower education) & $30363(84.1)$ & $759(81.1)$ & & $4904(14.3)$ & $432(16.9)$ & \\
\hline
\end{tabular}




\begin{tabular}{|l|c|c|c|c|c|c|}
\hline$<20$ (higher education) & $5228(14.5)$ & $153(16.3)$ & & $28829(84.3)$ & $2059(80.6)$ & \\
\hline N/A & $498(1.4)$ & $24(2.6)$ & & $451(1.3)$ & $63(2.5)$ & \\
\hline
\end{tabular}

"Education: Patient's measure of education was estimated by their area of residence by zip code and the proportion of adults in that zip code that did not graduate from high school from 2008-2012. N/A: not available; SD: standard deviation. 
Preoperative patient variables associated with increased mortality post-RC for UC

\begin{tabular}{|c|c|c|c|c|}
\hline & \multicolumn{2}{|c|}{ 30-day mortality } & \multicolumn{2}{|c|}{ 90-day mortality } \\
\hline & $\begin{array}{l}\text { Odds ratio } \\
(95 \% \text { CI })\end{array}$ & $\mathbf{p}$ & $\begin{array}{l}\text { Odds ratio } \\
\text { (95\% CI) }\end{array}$ & $\mathbf{p}$ \\
\hline Age (per 10-year increase) & $1.05(1.04-1.06)$ & $<0.001$ & $1.05(1.04-1.05)$ & $<0.001$ \\
\hline Sex $($ ref $=$ male $)$ & $0.78(0.63-0.96)$ & 0.02 & $0.96(0.84-1.08)$ & 0.47 \\
\hline cT stage $($ ref $=\mathrm{pT} \leq 1)$ & & & & \\
\hline $\mathrm{cT} 2$ & $1.23(0.99-1.54)$ & 0.064 & $1.30(1.13-1.51)$ & 0.001 \\
\hline cT3 & $1.34(0.97-1.86)$ & 0.075 & $1.74(1.43-2.12)$ & $<0.001$ \\
\hline cT4 & $1.72(1.22-2.40)$ & 0.002 & $2.72(2.23-3.32)$ & $<0.001$ \\
\hline $\mathrm{cN}$ stage $(\mathrm{ref}=\mathrm{cN} 0)$ & & & & \\
\hline $\mathrm{cN1}$ & $1.13(0.80-1.60)$ & 0.493 & $1.87(1.56-2.24)$ & $<0.001$ \\
\hline $\mathrm{cNx}$ & $1.12(0.84-1.50)$ & 0.434 & $1.37(1.15-1.64)$ & $<0.001$ \\
\hline $\begin{array}{l}\text { Neoadjuvant chemotherapy } \\
\text { (ref=no NAC) }\end{array}$ & $0.53(0.39-0.72)$ & $<0.001$ & $0.75(0.64-0.88)$ & $<0.001$ \\
\hline Race (ref=Caucasian) & & & 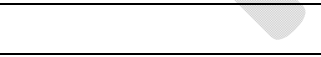 & \\
\hline African-American & $1.44(1.01-2.04)$ & 0.043 & $1.34(1.08-1.68)$ & 0.008 \\
\hline Other & $1.05(0.59-1.88)$ & 0.870 & $0.86(0.58-1.28)$ & 0.459 \\
\hline $\begin{array}{l}\text { Charlson-Deyo comorbidity } \\
\text { index (ref }=0)\end{array}$ & & & & \\
\hline 1 & $1.32(1.08-1.60)$ & 0.006 & $1.40(1.24-1.58)$ & $<0.001$ \\
\hline$\geq 2$ & $1.88(1.44-2.45)$ & $<0.001$ & $1.86(1.56-2.21)$ & $<0.001$ \\
\hline Income level (ref=low) & & & & \\
\hline High & $0.93(0.78-1.10)$ & 0.391 & $0.87(0.78-0.98)$ & 0.012 \\
\hline $\begin{array}{l}\text { Hospital volume per year } \\
(\text { ref } \leq 10)\end{array}$ & & & & \\
\hline $10-20$ & $0.85(0.67-1.07)$ & 0.165 & $0.94(0.81-1.08)$ & 0.319 \\
\hline$>20$ & $0.82(0.66-1.00)$ & 0.056 & $0.90(0.79-1.02)$ & 0.038 \\
\hline $\begin{array}{l}\text { Insurance (ref }=\text { no } \\
\text { insurance) }\end{array}$ & (2) & & & \\
\hline Medicare & $0.94(0.48-1.83)$ & 0.865 & $0.71(0.49-1.02)$ & 0.055 \\
\hline Medicaid & $0.71(0.30-1.66)$ & 0.428 & $0.94(0.61-1.45)$ & 0.751 \\
\hline Private & $0.69(0.36-1.35)$ & 0.281 & $0.58(0.40-0.84)$ & 0.003 \\
\hline Other government & $0.57(0.15-2.12)$ & 0.404 & $0.71(0.37-1.38)$ & 0.284 \\
\hline
\end{tabular}

$\mathrm{CI}$ : confidence interval; ref: reference value used for multivariable analysis 